\title{
An optimization method for nacelle design
}

\author{
Matthew Robinson ${ }^{1}$, David G. MacManus ${ }^{2}$, Alexander Heidebrecht ${ }^{3}$ \\ Cranfield University, Cranfield, United Kingdom, MK43 OAL \\ and \\ Nicholas Grech ${ }^{4}$ \\ Rolls-Royce plc, P.O Box 31 \\ Derby, United Kingdom, DE24 8BJ
}

\begin{abstract}
A multi-objective optimisation method is demonstrated using an evolutionary genetic algorithm. The applicability of this method to preliminary nacelle design is demonstrated by coupling it with a response surface model of a wide range of nacelle designs. These designs were modelled using computational fluid dynamics and a Kriging interpolation was carried out on the results. The NSGA-II algorithm was tested and verified on established multidimensional problems. Optimisation on the nacelle model provided 3-dimensional Pareto surfaces of optimal designs at both cruise and off-design conditions. In setting up this methodology several adaptations to the basic NSGA-II algorithm were tested including constraint handling, weighted objective functions and initial sample size. The influence of these operators is demonstrated in terms of the hypervolume of the determined Pareto set.
\end{abstract}

\section{Nomenclature}

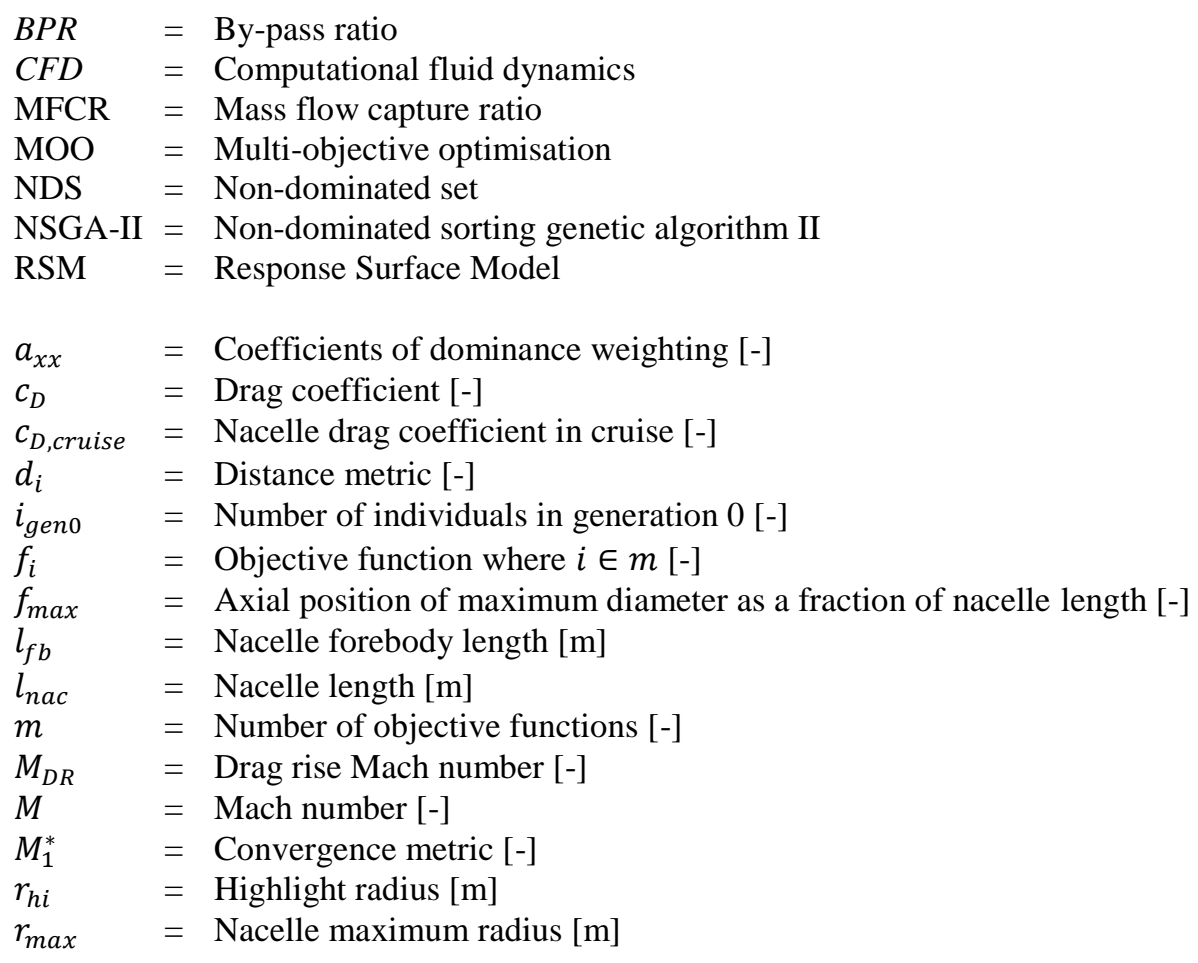

${ }^{1} \mathrm{PhD}$ Student Researcher, Propulsion Engineering Centre, Cranfield University.

${ }^{2}$ Head of Gas Turbine Technology Group, Propulsion Engineering Centre, Cranfield University, AIAA Member.

${ }^{3}$ Research Fellow, Propulsion Engineering Centre, Cranfield University

${ }^{4}$ Aerothermal Engineer, Installation Aerodynamics, Rolls Royce plc.

American Institute of Aeronautics and Astronautics 


$\begin{array}{ll}r_{T E} & =\text { Trailing edge radius }[\mathrm{m}] \\ r_{i f} & =\text { Initial forebody radius }[\mathrm{m}] \\ \mathrm{P}_{\mathrm{t}} & =\text { Parent population [-] } \\ \mathrm{Q}_{\mathrm{t}} & =\text { Child population [-] } \\ \mathrm{x}_{\mathrm{i}} & =\text { Decision variable [-] } \\ Y^{\prime} & =\text { Optimal set from optimisation [-] } \\ \bar{Y} & =\text { Pareto set [-] } \\ w_{k}^{\prime} & =\text { Coefficients of spacing weighting [-] } \\ \beta_{n a c} & =\text { Nacelle boat tail angle }\left[{ }^{\circ}\right] \\ \Delta & =\text { Diversity uniformity metric [-] } \\ \Delta c_{D, \text { spill }} & =\text { Change in spillage drag coefficient [-] } \\ \sigma & =\text { Standard deviation } \\ \Omega_{i} & =\text { Modified objective function }[-]\end{array}$

\section{Introduction}

Wistorically the development of the aero-engine has followed a trend of increasing bypass ratio (BPR) to improve propulsive efficiency [1]. As the bypass ratio has increased the fan diameter has grown to much larger sizes, whilst the relative size of the core size has reduced [2]. The limitation to this approach for underwing configurations could potentially arise from an excessive engine installation penalty due to nacelle wetted area and adverse wing interference A previous study reported an overall nacelle contribution of around $14 \%$ of the drag for a civil twin-engine airliner [3]. With much larger engines mounted under the wing, the impact of interference effects on the wing increases and correct engine sizing and placement is of greater importance [4]. The penalty from a larger diameter engine nacelle is apparent as an increase in overall aircraft drag and weight. The drag is caused by a larger nacelle wetted area which incurs additional skin friction and an increase in installation drag. For example, one study [5] found that whilst an increase in BPR from 11.5 to 21.5 reduced the specific fuel consumption by $7 \%$, the optimal engine in terms of block fuel burn was $\mathrm{BPR}=14.3$. This was a result of the installation of the larger engine incurring weight, drag and interference penalties. Therefore in order to avoid excessive installation penalties associated with the larger engine diameter, these aspects need to be addressed.

To assess the plausibility of larger engines, an assessment of the nacelle performance is required at a preliminary stage in the design process. The definition of a nacelle is an engineering problem which must incorporate requirements from different operating conditions and design points. The design can therefore be viewed as a multiobjective problem and previous work has been carried out to optimise the aerodynamic shape [6] [7]. To reduce the computational cost previous work has looked at optimisation based on surrogate models such as a Kriging interpolation of a design space [6]. This surrogate modelling allows quick assessment of a nacelle design in a Pareto front and allows an optimisation to be carried out in a much smaller amount of time in comparison to a higher order simulation.

Of specific interest in the aerodynamic design of nacelles is the drag during the cruise portion of the flight since, in general, this will contribute the most to the overall fuel burn. However it is also important that the nacelle performs well in off-design conditions. These include operation at a range of flight Mach numbers and engine mass flows. When an aero-engine nacelle operates in the transonic region it is important to have knowledge of the drag rise Mach number $\left(M_{D R}\right)$, that is the value of freestream Mach number at which a precipitous rise in drag occurs. In addition to the drag rise, the influence of the nacelle design on the spillage drag is also important. Spillage drag is incurred when the ratio of the freestream stream tube area is less than the area of the nacelle highlight. This area ratio is called the mass flow capture ratio (MFCR) and typically in cruise a nacelle will operate at around MFCR=0.7 [8].

Multi-objective optimisation (MOO) deals with a certain class of mathematical problems in which a 'Pareto' surface of trade-offs is sought [9]. Since a balance of performance at various operating points is required for nacelle design, a Pareto optimal set must be found. A certain class of methods used to determine a Pareto surface in a MOO problem, termed evolutionary algorithms, mimic evolution in nature. These evolutionary algorithms have the benefit that they can create and preserve diversity across the solutions by simulating a population of individuals in each iteration. The aim of this paper is to demonstrate an application of an evolutionary algorithm to nacelle design and to assess how efficiently this can be performed for the purposes of preliminary nacelle design. Multi-objective optimisation is utilised to demonstrate the need to concurrently optimise for more than one performance metric. A method is then demonstrated which can be used to understand such nacelle performance Pareto fronts. 


\section{Methods and scope}

\section{A. Nacelle definition and parameterisation}

To allow preliminary design optimisation, a rapid prediction of nacelle performance based on geometric and aerodynamic parameters is required. A nacelle parameterisation was developed in a previous study [10] which consisted of 7 design variables (Figure 1). The axial end points of the nacelle are defined by $l_{n a c}, r_{h i}$ and $r_{T E}$, of which $r_{T E}$ is typically set by a required nozzle area, $r_{h i}$ is defined by the intake design and the $l_{n a c}$ is reduced insofar as is practicable. The forebody length $\left(l_{f b}\right)$ and initial forebody radius $\left(r_{i f}\right)$ determine the shape and curvature distribution of the forebody and therefore the aerodynamic loading. The boattail angle $(\beta)$ is then set to produce a steady diffusion from the maximum radius to the trailing edge. The nacelles were constructed from iCST curves [12] with a simplified cylindrical nozzle geometry (Figure 1). Class Shape Transformation (CST) curves consist of a class function which is modified by a shape function [11]. The class function can define several shapes including a straight line and an aerofoil with a curved leading edge and a sharp trailing edge. iCST curves are an adaptation of CST which solve the underlying equations through the specification of 'intuitive' variables.

Two aerodynamic variables of particular interest in nacelle design are the freestream Mach number $\left(M_{\infty}\right)$ and the MFCR. Through the assessment of the drag as a function of $M_{\infty}$ and MFCR, the drag rise Mach number and the spillage drag can be assessed. To understand the influence on the drag from the six geometric variables (Figure 1) and the Mach number $\left(M_{\infty}\right)$ and MFCR, a lower order model was desired. To have an appropriate representation of the drag gradients as a function of the $M_{\infty}$ and MFCR, a large number of individual simulations are required. In addition, a three level full factorial assessment of the geometric variables would consist of $3^{6}$ designs, each of which would require in the order of at least hundred aerodynamic simulations to determine the performance. A response surface model (RSM) has been developed from computational fluid dynamics simulations of a wide variety of axisymmetric nacelle designs. The RSM consisted of a design space of 8 dimensions including 6 geometric variables $\left(l_{n a c}, r_{\max }, r_{T E}, l_{f b}, r_{i f}, \beta\right)$ (Figure 1) [10] and 2 aerodynamic variables $\left(M_{\infty}, M F C R\right)$ with modified nacelle drag [10] as the dependant variable. A design of experiments was constructed for 153 nacelle designs with a Latin hypercube approach.

The computational analyses for these nacelle designs were represented with a simplified cylindrical nozzle (Figure 1). These configurations used an axisymmetric multi-block mesh with a semi-circular domain which incorporated a farfield boundary condition [10]. A mesh independence study was carried out using three meshes and a mesh size of approximately 39000 used for each axisymmetric design [10]. Similarly a domain sensitivity study found that the nacelle drag varied less than $0.004 \%$ from an increase in domain radius from 70 times the maximum nacelle radius to 80 times the maximum nacelle radius [10]. The domain was therefore set to 80 times the nacelle maximum radius [10]. The total pressure and total temperature at the inlet to the nozzle were specified to equal the freestream value which acts to remove the influence of the post exit stream tube. Each of these 153 designs was simulated across 224 aerodynamic points with a steady RANS computational fluid dynamics (CFD) method. The CFD analyses were performed using an implicit density-based solver with a $2^{\text {nd }}$ order discretisation scheme. This covered a range of $M_{\infty}$ from 0.2 to 0.95 and a range of MFCR from 0.3 to $95 \%$ of the intake choking mass flow.

The RSM was created from a Kriging interpolation of the results of all the aerodynamic analyses. Consequently this enables the calculation of the drag rise Mach number [13] $\left(\mathrm{M}_{\mathrm{DR}}\right.$, equation (1)) and a nominal spillage drag $\left(\Delta c_{D, \text { spill }}\right.$, equation (2)) for each design within the RSM. These designs were analysed across a range of aerodynamic points and the RSM was then coupled with an optimisation method for rapid assessment of nacelle drag as a function of the geometry.

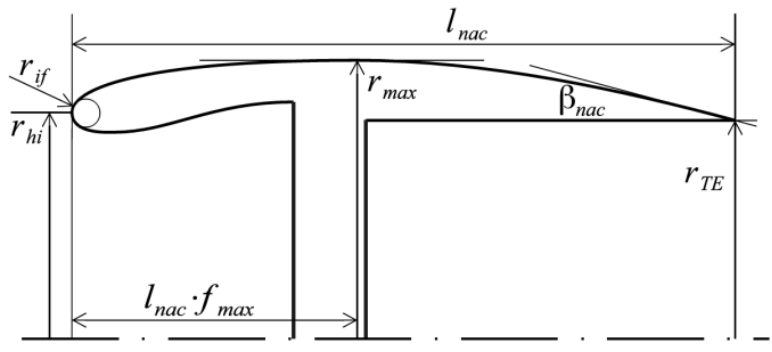

Figure 1. Geometric parameters used to construct RSM [7] 


$$
\begin{gathered}
\left.\frac{\partial C_{D}}{\partial M_{\infty}}\right|_{\mathrm{M}=\mathrm{M}_{\mathrm{DR}}}=0.05 \\
\Delta c_{D, \text { spill }}=c_{D, M F C R=0.65}-c_{D, M F C R=0.7}
\end{gathered}
$$

\section{B. Optimisation method}

To address the need of rapid design for engine nacelles an optimisation tool has been developed which can determine the optimal fitness for a given set of objectives in a search space. The non-dominated sorting genetic algorithm II (NSGA-II) was proposed by Deb [14] as an appropriate evolutionary algorithm for a wide variety of multi-objective problems. The algorithm is a multi-step process which aims to find the optimal fitness for a given set of objectives. The concept of dominance is used to rank fitness levels of individuals where an individual (x) dominates another (y) if it is no worse in any objective function and strictly lower in at least one (equation (3)). The NSGA-II algorithm starts by creating a combined population from a parent population $\mathrm{P}_{\mathrm{t}}$ and a child population $\mathrm{Q}_{\mathrm{t}}$. This combined population is then sorted into non-dominated sets (NDSs), in which no individual dominates any other. Within these NDSs, individuals are given a crowd distance value based on their proximity to other solutions in the NDS. Tournaments are then carried out between individuals to determine the subsequent mating pool from which later generations are produced as part of the optimisation process. The subsequent generation utilises this mating pool along with crossover and mutation to ensure variation. This algorithm has been developed to preserve diversity, whilst quickly converging on a Pareto optimal front and has been demonstrated as appropriate for several non-convex problems [15].

$$
f_{i}(x) \leq f_{i}(y)
$$

Within this work an optimisation routine has been established based on the NSGA-II evolutionary algorithm (Figure 2). This routine begins with a randomly generated vector which creates the first generation of individuals. These are then dimensionalised based on the bounds of the degrees of freedom used to define the edges of the desired design space. Corresponding objective values are then evaluated from either the benchmark function or from an RSM interpolation. These values are ranked by dominance, distance and tournaments carried out. From the subsequent mating pool which is determined from these tournaments the next generation is produced using blend crossover (BLX) and Gaussian mutation. This overall process then repeats for subsequent generations. Unless otherwise stated all optimisation analyses were carried out with the settings in Table 1.

Table 1 Algorithm settings

\begin{tabular}{r|c}
\hline Parameter & Value \\
\hline Number of generations & 100 \\
Population size & 40 \\
Crossover rate & 0.1 \\
Mutation rate & 0.2 \\
Gaussian mutation $\sigma$ & 0.0005 \\
\hline
\end{tabular}




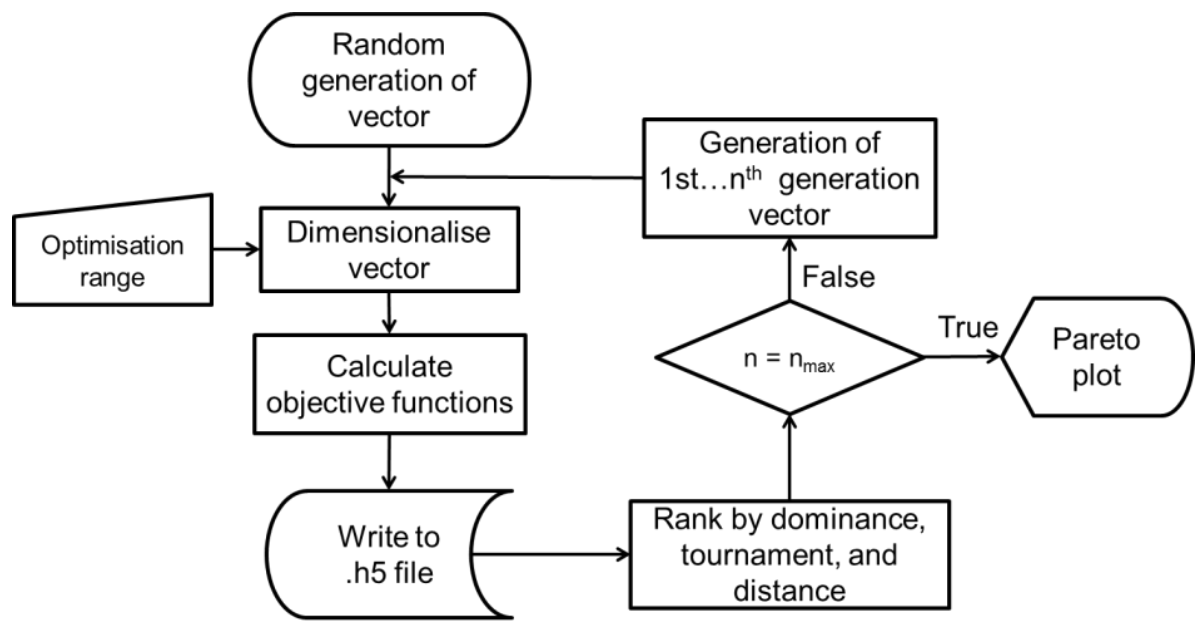

Figure 2. Optimisation routine outlined as a flow chart

A multi-objective-optimisation approach was applied to three objective functions. The design point for an engine nacelle is the cruise condition at which the drag should be minimised to generally reduce the fuel burn of an aircraft. Therefore the mid-cruise drag $\left(c_{D, \text { cruise }}\right)$ was assessed as the first objective function which for the considered nacelle was estimated at the aerodynamic operating point of $\mathrm{M}=0.85$, and a mass flow capture ratio (MFCR) of 0.7. In addition to performing as well as possible at this design point, it is also desirable that a nacelle should not invoke too high a drag penalty when operating off design. The drag rise Mach number at cruise MFCR $=0.7$ was used as the second objective function. Spillage was assessed as a $\Delta c_{D}$ between a mid-cruise MFCR and an end of cruise MFCR and this used as the third objective function. Five geometric degrees of freedom for the optimisation vector were used with the bounds given in Table 2. These are five variables which represent the design space available for an engine design with a prior knowledge of the trailing edge radius.

Table 2 Bounds used for the optimisation using the RSM

\begin{tabular}{c|ll}
\hline Parameter & Lower bound & Upper bound \\
\hline$l_{f b} / r_{h i}$ & 0.35 & 0.5 \\
$r_{\max } / r_{h i}$ & 1.15 & 1.35 \\
$\beta_{\text {nac }}$ & 10 & 15 \\
$l_{\text {nac }}$ & 5.57 & 8.36 \\
$r_{i f} l_{f b} /\left(r_{\text {max }}-r_{h i}\right)^{2}$ & 0.6 & 1.2 \\
\hline
\end{tabular}

\section{Performance metrics}

The ability of an algorithm to effectively solve an MOO problem relies on its ability to both converge on the optimal set of solutions and to avoid clustering around a specific non-dominated solution. Performance, therefore, of an algorithm can be assessed in terms of proximity of solutions to the true Pareto set and diversity of solutions in optimisation. To give an indication of the relative performances of the analyses carried out, three metrics are considered in this paper. Each of these gives slightly different information on the convergence and/or diversity of the final optimised Pareto set. Zitzler [16] defined the metric $M_{1}^{*}$ (equation (6)) to assess the performance of an algorithm for the proximity of solutions to the Pareto set and Deb proposed a metric $\Delta$ (equation (7)) for the diversity of solutions in optimisation. The convergence metric $\left(M_{1}^{*}\right)$ is calculated for an optimal set found from optimisation $\left(Y^{\prime}\right)$ by comparison to a known Pareto front $(\bar{Y})$. The Euclidean distance $\left(\left\|d^{\prime}-\bar{d}\right\|\right)$ from the true Pareto front $(\bar{d})$ is assessed for each member of the optimal set $\left(d^{\prime}\right)$. An optimal set which has converged perfectly to the Pareto set will have a value of $M_{1}^{*}=0$ [16]. The diversity parameter relates the difference between a distance metric $\left(d_{i}\right)$ and the average distance metric $(\bar{d})$ for the entire optimal set $\left(Y^{\prime}\right)$ of size $\mathrm{N}$. The distance metric used in this work was the crowding distance as suggested for the NSGA-II algorithm [14]. $d_{f}$ and $d_{l}$ correspond to the distance to the extreme values of the Pareto set. An optimally spread out solution will have a diversity metric of $\Delta=$ 0 since the distance between every individual $\left(d_{i}\right)$ would be equal to the average $(\bar{d})$ and no extremities would exist 
$\left(d_{f}, d_{l}\right)$ [14]. Since the true Pareto front of the Kursawe function [17] is an unknown, $M_{1}^{*}$ cannot be assessed for this test case, however $\Delta$ can be assessed to determine the ability to maintain diversity within the optimal solutions.

$$
\begin{gathered}
f_{1}(x)=\sum_{i=1}^{2}\left[-10 \exp \left(-0.2 \sqrt{x_{i}^{2}+x_{i+1}^{2}}\right)\right] \\
f_{2}(x)=\sum_{i=1}^{3}\left[\left|x_{i}\right|^{0.8}+5 \sin \left(x_{i}^{3}\right)\right] \\
M_{1}^{*}=\frac{1}{\left|Y^{\prime}\right|} \sum_{d \prime \in Y \prime} \min \left\{\left\|d^{\prime}-\bar{d}\right\|^{*} ; \bar{d} \in \bar{Y}\right\} \\
\Delta=\frac{d_{f}+d_{l}+\sum_{i=1}^{N-1}\left|d_{i}-\bar{d}\right|}{d_{f}+d_{l}+(N-1) \bar{d}}
\end{gathered}
$$

The hypervolume [18] is another metric which combines the convergence and the diversity into a single value (equation (8)). The hypervolume of a solution set $\left(Y^{\prime}\right)$ is defined as the m-dimensional space which is covered by the set in reference to an arbitrary point (Figure 3). If the reference point is greater in value than the population individuals, and the objectives are to be minimised, then higher values of hypervolume represent improvements in the solution space. The hypervolume is increased by both improvements in convergence and diversity. A benefit of this metric is that it can also be compared to the hypervolume of a known Pareto set $(\bar{Y})$ to determine the percentage of the true Pareto set which has been found by the algorithm.

$$
H V=\operatorname{volume}\left(\mathrm{U}_{i=1}^{\left|Y^{\prime}\right|} v_{i}\right)
$$

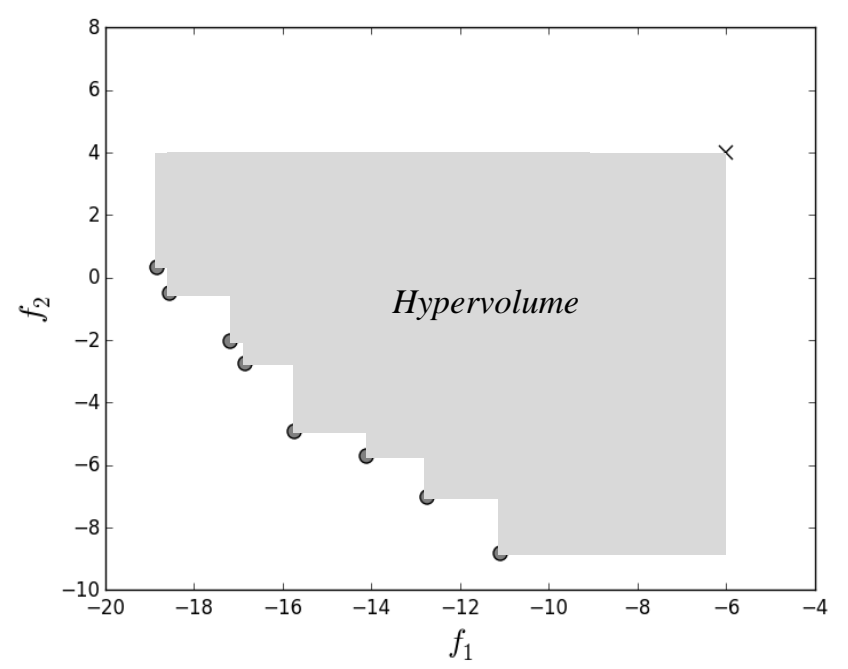

Figure 3. Schematic of hypervolume in two dimensions, based on [19] 


\section{Results and analysis}

\section{A. Benchmark test cases}

To verify that the NSGA-II algorithm has correctly been implemented and that it can correctly determine optimal sets in a variety of problems, two benchmark functions were tested.

\section{Kursawe test function}

The Kursawe function [17] is a two objective problem with a disconnected Pareto front which adds to the complexity in acquiring a distribution along the entire front. It involves minimisation of two functions (equations (4) and (5)) using three degrees of freedom each of which is bounded between -5 and 5 . The optimisation tool was applied using a generation size of 40 for 100 generations. The convergence of the optimisation process was assessed based on the evolution of the hypervolume throughout the generations. This analysis demonstrated a monotonic progression and that beyond generation 61 there is less than $1 \%$ increase in the hypervolume relative to the initial hypervolume indicating that the solution is converged.

The results from this analysis (Figure 4) show that a spread in $\mathrm{f}_{1}$ was achieved between -19.95 and -14.40 whilst $\mathrm{f}_{2}$ varied between -11.52 and 0.06 . These ranges are similar to those found by NSGA-II previously [14] and overall the optimisation covered all the disconnected regions found in previous analyses [20] and had a diversity of $\Delta=0.531$. The Kursawe function is a standard test case for MOO algorithms and these results demonstrate the ability of this routine to find the disconnected Pareto fronts.

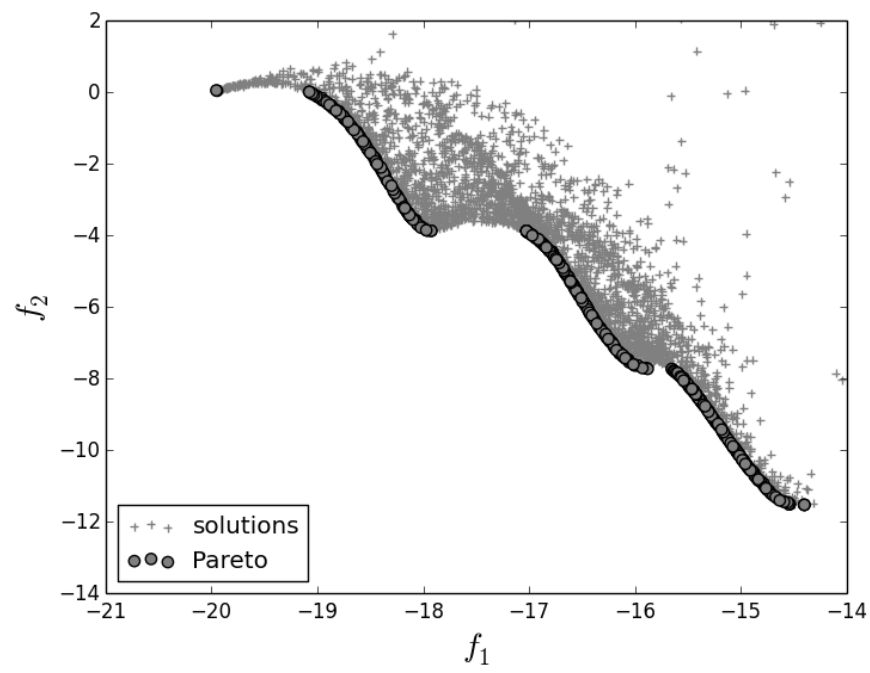

Figure 4. Evaluations of the Kursawe function [17] plotted in objective space showing the Pareto front

The hypervolume was determined for 40 independent instances of the optimisation tool to assess statistically how reliable the implementation was. There are some outliers in the sample solutions with two lying beyond 3 standard deviations from the mean hypervolume. Given that the implementation of the NSGA-II was identical between the optimisation instances these differences arise from the nondeterminism of the algorithm. Three stages of the algorithm incorporate randomness. The first of these is the initial generation in which random vectors initialise the algorithm. The second and third are the mutation and crossover operators which include a random number and are randomly applied according to a specific rate. To investigate the calibration of this randomness additional tests were carried out with the Kursawe function (Figure 5). Further optimisation analyses were performed with an enlarged zeroth generation size $\left(i_{\text {gen } 0}=400\right)$, but with the same number of evaluations of 4000 . This enlarged zeroth generation led to an improved initial set from which the optimisation process was initiated (Figure 5) with a higher median hypervolume in the 40 instances of the optimisation. It also had less scatter with a range of 53.6 compared to 89.4 from the standard $i_{\text {gen } 0}=40$. This ultimately gave a similar median and interquartile range (IQR) for the final generation $n_{g e n}=N$ for both configurations. However, there were fewer outliers in the configuration with an enlarged zeroth generation suggesting a more robust implementation for the same number of evaluations. This therefore indicates that an enlarged random sample may be of use to improve the likelihood of convergence to the Pareto front without any increase in computational cost. 


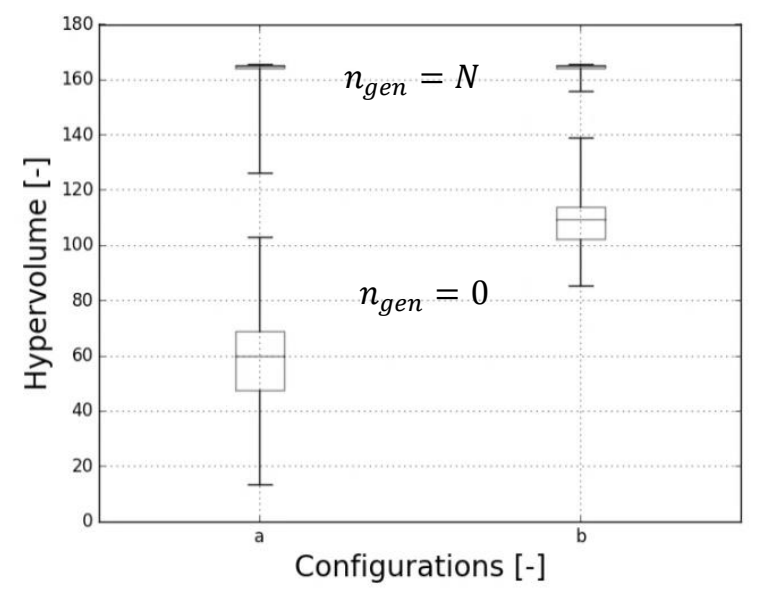

Figure 5. Median hypervolume of zeroth and final generations with IQR and range for 40 optimisation analyses of the Kursawe function with (a) $i_{\text {gen } 0}=40, N=100$ and (b) $i_{\text {gen } 0}=400, N=90$

\section{DTLZ2}

The DTLZ2 test problem was proposed to be scalable to the required number of degrees of freedom and objective functions [15]. The optimisation tool was applied to a formulation of this problem which consists of 5 variables and 3 objectives (equations (9),(10),(11)) to be representative of the three objectives which will be used for nacelle optimisation. Results from this demonstrate that optimal individuals over the three objectives was obtained by using a population size of 40 over 100 generations (Figure 6). To determine the performance of the algorithm with 3 objective functions, the optimal solutions were compared to the analytical Pareto set (sphere of radius 1) to calculate $M_{1}^{*}$ and $\Delta$. These metrics suggest a close approximation of the true Pareto front with a convergence of $M_{1}^{*}=0.0109$ with a relatively even diversity of $\Delta=0.438$. A previous study with NSGA-II on the DTLZ2 problem indicated values in the range of $\Delta=0.5$ and $\Delta=0.8$ with an average performance around $\Delta=0.7$ [21]. This gave confidence that the NSGA-II algorithm had been implemented correctly and that the parameters for the mutation and crossover were well posed.

$$
\begin{aligned}
& f_{1}(x)=\left(1+g\left(\boldsymbol{X}_{M}\right)\right) \cos \left(\frac{x_{1} \pi}{2}\right) \cos \left(\frac{x_{2} \pi}{2}\right) \\
& f_{2}(x)=\left(1+g\left(\boldsymbol{X}_{M}\right)\right) \cos \left(\frac{x_{1} \pi}{2}\right) \sin \left(\frac{x_{2} \pi}{2}\right) \\
& \quad f_{3}(x)=\left(1+g\left(\boldsymbol{X}_{M}\right)\right) \sin \left(\frac{x_{1} \pi}{2}\right) \\
& \text { Where } g\left(\boldsymbol{X}_{M}\right)=\sum_{x_{i} \in X_{M}}\left(x_{i}-0.5\right)^{2} \text { for } x_{3}, x_{4}, x_{5} \in \boldsymbol{X}_{M}
\end{aligned}
$$

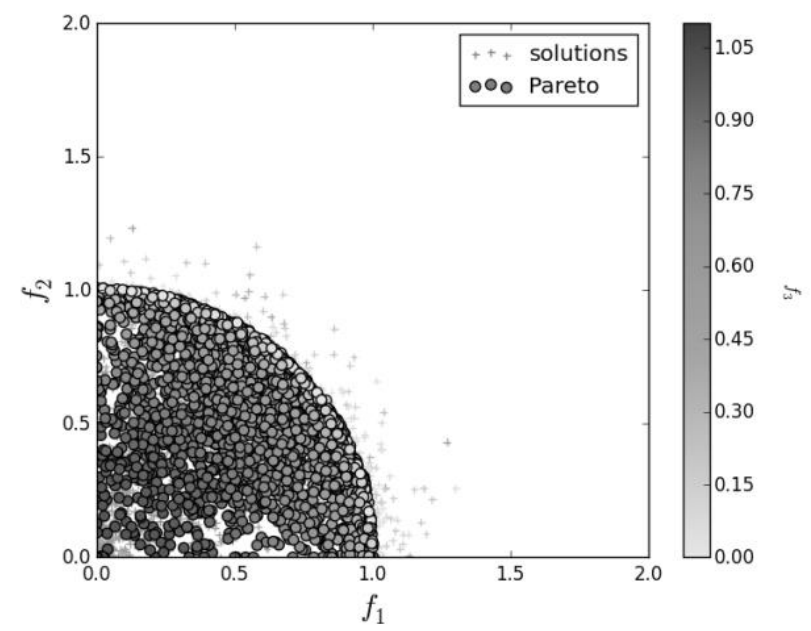

Figure 6. Evaluations of the DTLZ2 function [13] plotted in objective space showing the Pareto front 
Hypervolume can be calculated exactly for the DTLZ2 problem since the Pareto set $(\bar{Y})$ is known. As such, the performance of the NSGA-II algorithm can be assessed in terms of percentage of the true Pareto front which is determined. The hypervolume was assessed from the reference coordinate of $(2,2,2)$. The hypervolume covered by the NSGA-II algorithm $\left(Y^{\prime}\right)$ reached $99 \%$ of the true hypervolume $(\bar{Y})$ within 30 generations; after 100 generations the hypervolume was $99.6 \%$ (Figure 7a). A good MOO method should avoid bias towards any of the objective functions. The three objectives in the DTLZ2 function were individually assessed to determine the convergence in each dimension of the search space. The mean value in each of the dimensions is 0.5 for a perfectly converged solution. The results of this optimisation converged to within $10 \%$ of 0.5 for all three objective functions after 47 generations (Figure 7b). No objective function reached a significantly different level of convergence. Similar levels of hypervolume convergence for NSGA-II has been reported for the DTLZ2 problem [22].
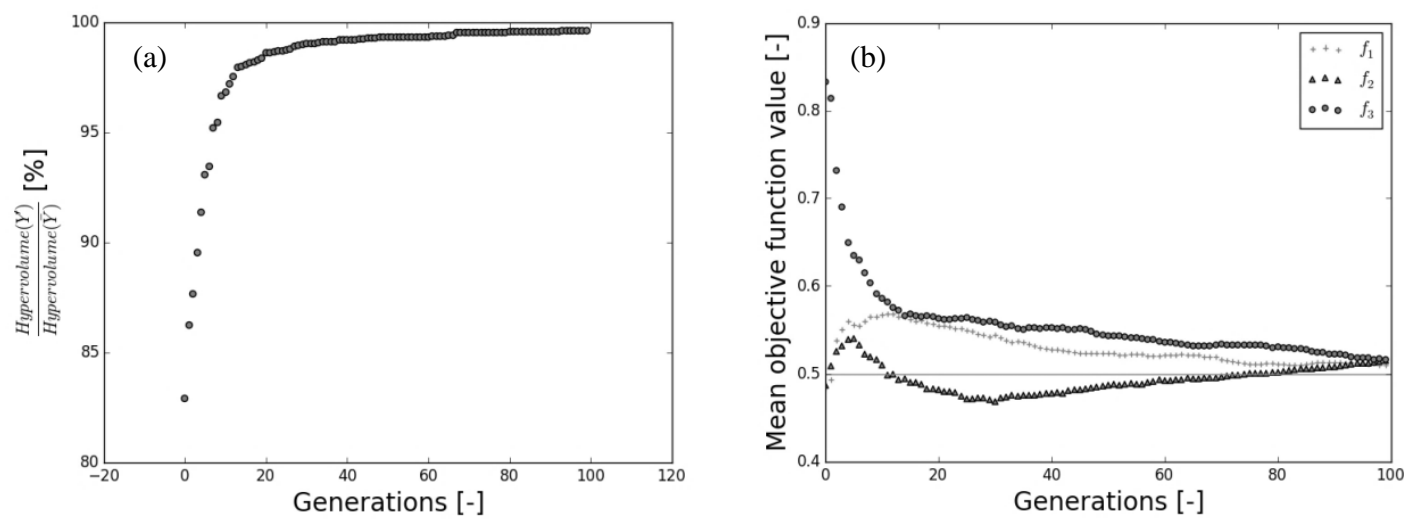

Figure 7. As a function of the generation number for the DTLZ2 function [15] evaluated over 100 generations: (a) Percentage of true Pareto hypervolume (b) Mean objective function values

\section{B. Optimisation on a nacelle drag response surface model}

The optimisation routine described above was applied to the RSM of the nacelle aerodynamic characteristics. The aim was to determine the Pareto optimal solutions within the Kriging interpolation for the three objective functions defined $\left(c_{D, \text { cruise }}, M_{D R}, \Delta c_{D, \text { spill }}\right)$. The optimisation on the RSM began with a random initial generation (Figure 8a) which was then used to produce subsequent, improved generations (Figure 8b,c,d). After 100 generations and 4000 individual evaluations of the RSM model the non-dominated members of these generations produce an overall optimal Pareto front $\left(Y^{\prime}\right)$ (Figure 9). It can be seen from this optimal set that the design space narrows as the drag rise Mach number is improved until $\mathrm{M}_{\mathrm{DR}}=0.896$, above which there are no nacelle designs which have been identified in the optimal set $\left(Y^{\prime}\right)$. To obtain drag rise Mach numbers above $\mathrm{M}_{\mathrm{DR}}=0.89$ spillage drag increases to above $\Delta c_{D, \text { spill }}=0.0028$, however this can be traded off against the cruise drag. Overall, the optimisation produced a relatively large tradeoff surface from which designs could be chosen. A designer will typically design for a $M_{D R}$ which is 0.02 above the desired cruise Mach number [23] and as such this tradeoff surface allows cruise Mach numbers in excess of $M=0.87$. The designer could then choose from amongst the designs one which gave the lowest overall fuel burn integrated over a full mission. Relative to lowest drag found in the random design space search (Figure 8a) the final optimal designs have up to a 7\% reduction in drag. The final Pareto had a diversity measure of $\Delta=0.449$. This value provides a baseline for the alterations to the methodology tested in the next section. 

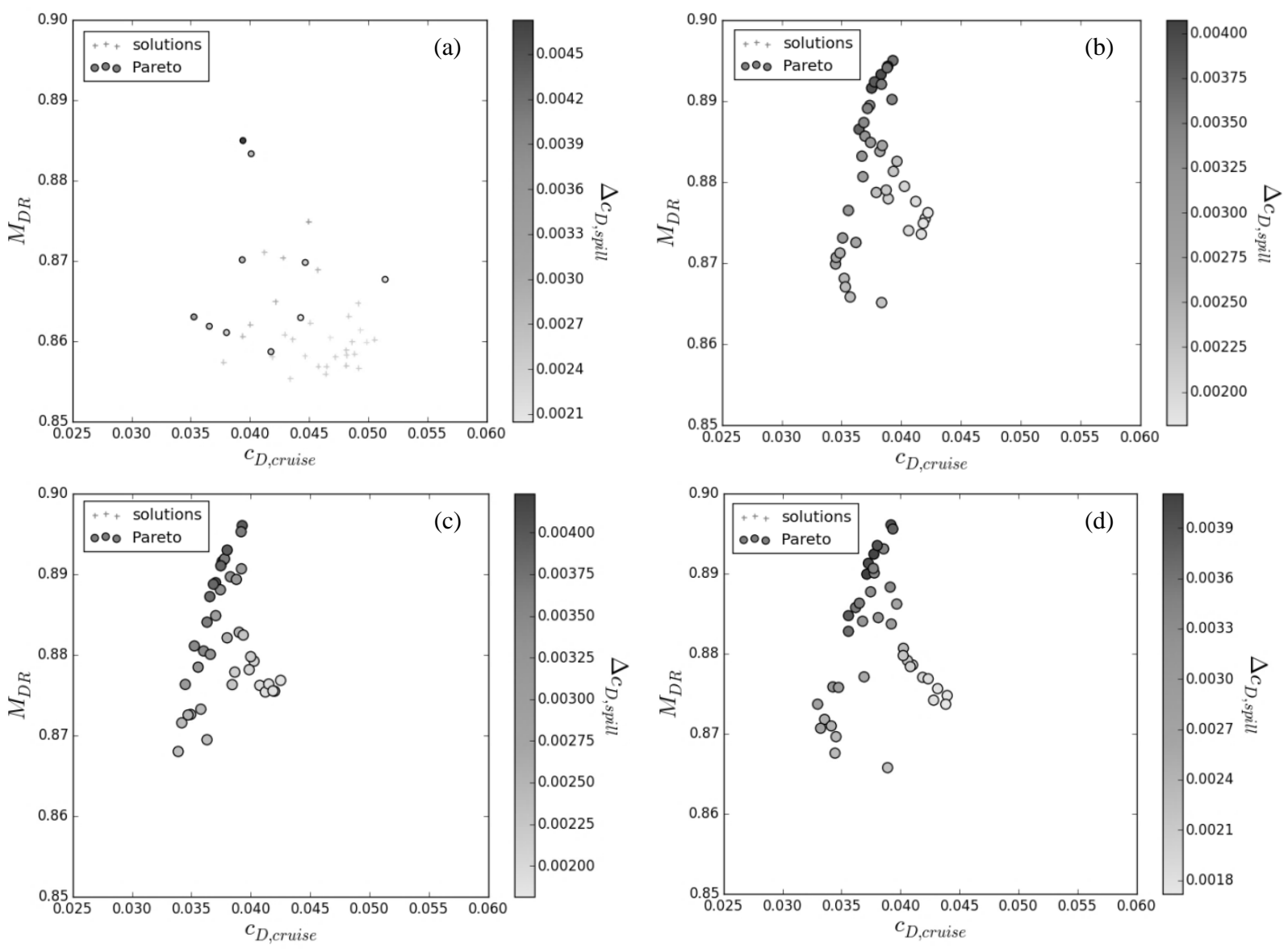

Figure 8. Population in objective space for (a) generation 0 (b) generation 25 (c) generation 50 and (d) generation 99

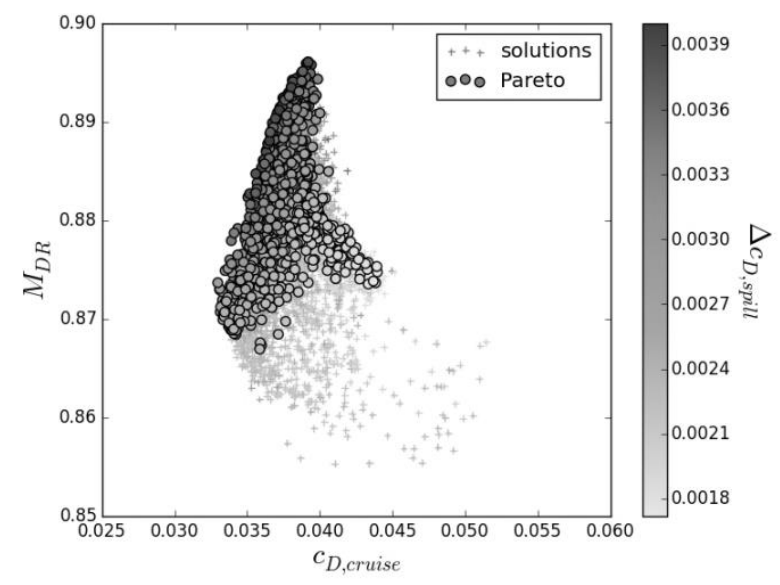

Figure 9. Final Pareto optimal set extracted from the RSM plotted as a function of the 3 objective functions

\section{Influence of initial sample size}

The design space for nacelle performance in terms of the three functions $\left(c_{D, \text { cruise }}, M_{D R}, \Delta c_{D, \text { spill }}\right)$ is expected to have an uneven shape due to different viable designs producing effective local minima. Consequently it is important to ensure that the implementation of the NSGA-II optimisation algorithm is sufficiently calibrated to ensure correct convergence and diversity preservation since the MOO problem of nacelle design may not be represented fully by 
existing benchmark functions. To determine the dependency of the method on the initial estimate from the random sample, the initial population size was varied. To perform well, an algorithm should be independent of the random sample and should converge to a Pareto set of reasonably equivalent fitness irrespective of the initial data. The results from this analysis showed that using different initial sample sizes could have some influence over the final optimal designs (Figure 10). The peak $\mathrm{M}_{\mathrm{DR}}$ achieved is mostly invariant at about 0.896, but with a larger initial sample (Figure 10b,c,d) a greater range of Pareto solutions is present at lower $\mathrm{M}_{\mathrm{DR}}$ numbers, covering up to a $30 \%$ wider range in terms of drag coefficient $\left(c_{D, \text { cruise }}\right)$ and up to a $1.5 \%$ wider range in the spillage drag $\left(\Delta c_{D, \text { spill }}\right)$. The diversity metric of the solutions is not highly influenced by the initial seed however with a minimum value from an initial population size of $i_{g e n 0}=200$ of $\Delta=0.419$, which does not offer a significant improvement from $\Delta=0.449$ after 100 generations.
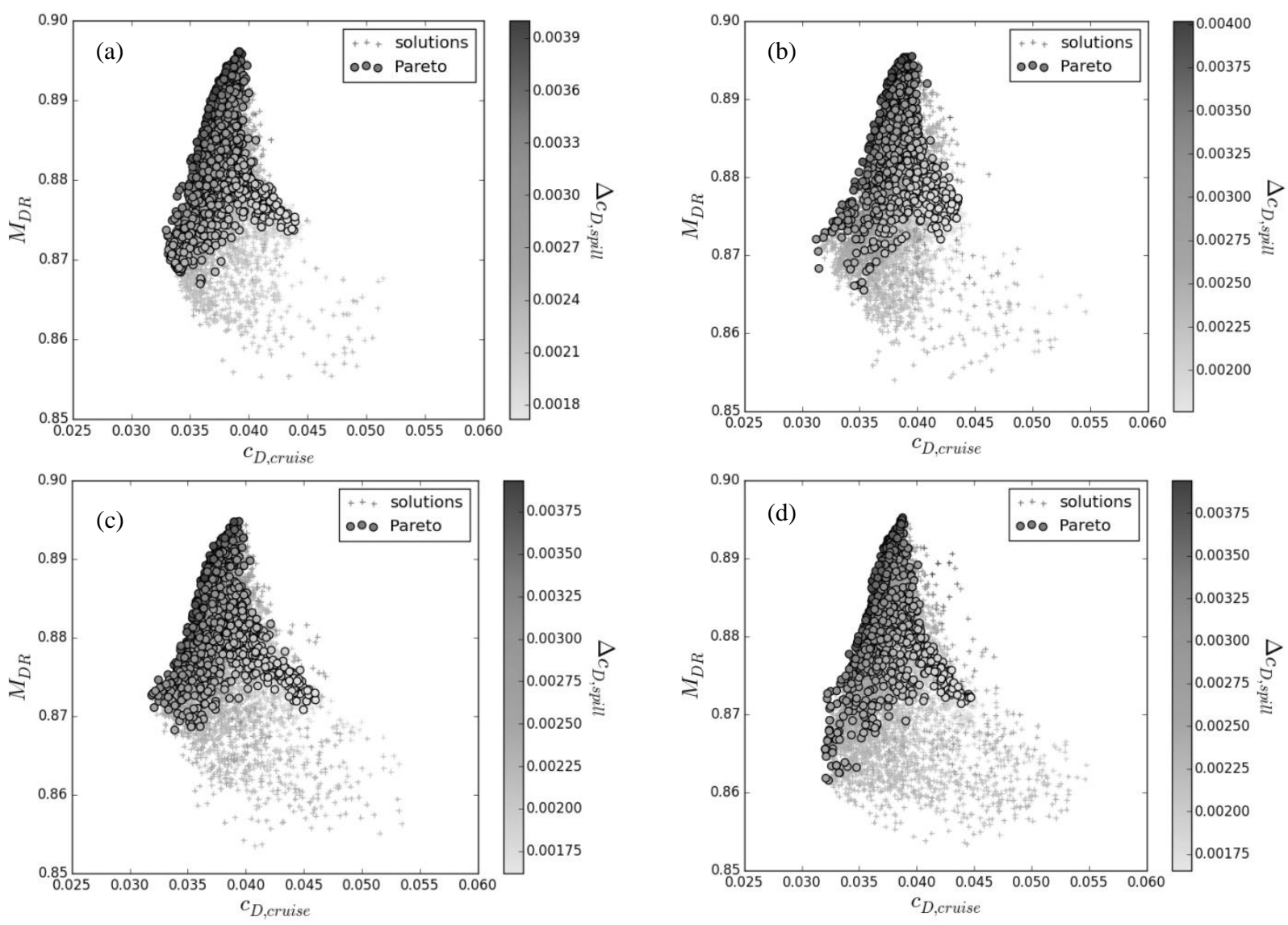

Figure 10. Final Pareto optimal set extracted from the RSM with (a) $i_{g e n 0}=40$ (b) $i_{g e n 0}=100$ (c) $i_{\text {gen } 0}=200(\mathrm{~d}) i_{\text {gen } 0}=400$

A potential benefit from using a larger initial random generation may come from reducing the number of overall generations. Whilst solving simple benchmark functions or extracting interpolated values from an existing RSM are relatively quick to evaluate, this method could be applied to more time consuming evaluations such as CFD and in this instance reducing the number of evaluations could equate to significant resource savings. Figure 11 demonstrates the influence of using an initial seed with a relatively low number of generations. To ensure a fair comparison, two computations have been computed over 1200 evaluations where the optimisation with $i_{\text {gen } 0}=40$ has been computed for 30 generations and the analysis with $i_{\text {gen } 0}=400$ covers 20 generations. The optimisation with the larger initial sample produced a Pareto front with a greater, more uniform spread, $\Delta=0.399$ compared to $\Delta=0.414$. This allowed a wider range of individuals in the trade-off surface between $c_{D, n a c}$ and $\Delta c_{D, \text { spill }}$ with lower 
values achieved in both. Specifically a reduction in the $c_{D, \text { cruise }}$ of $3 \%$ and $6 \%$ in $c_{D, \text { spill }}$ was found by use of an enlarged $i_{\text {gen } 0}$.
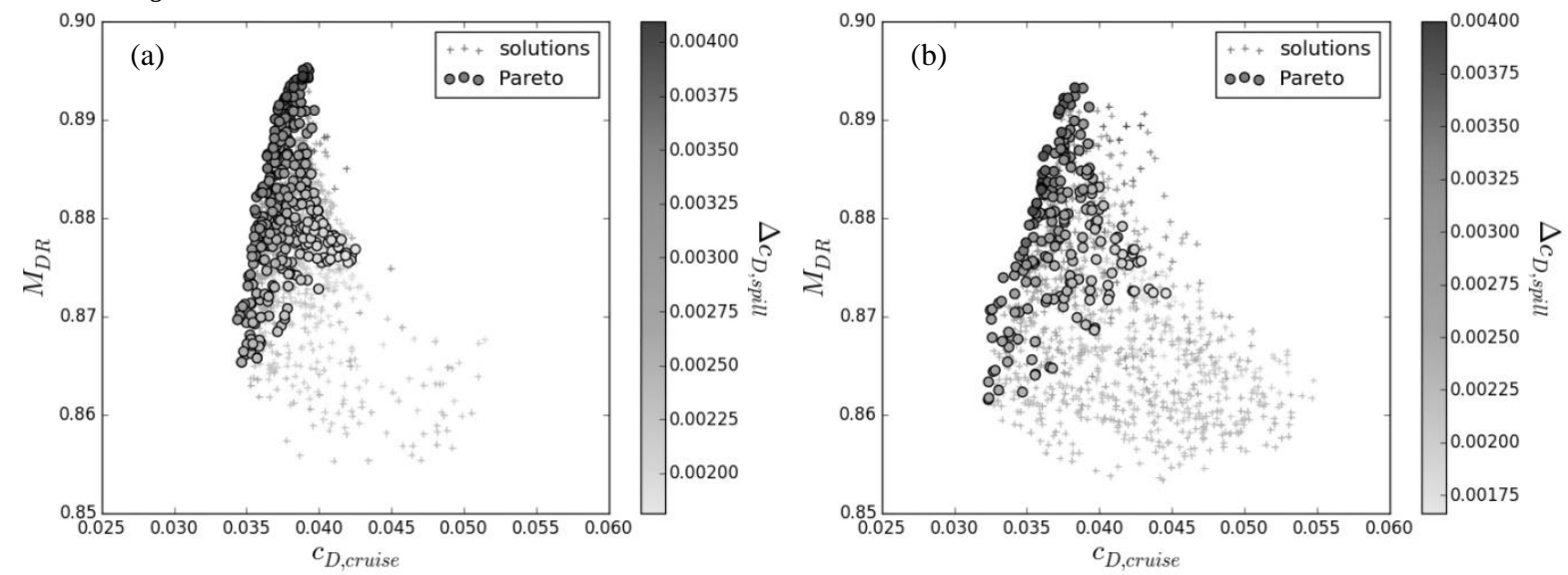

Figure 11. Final Pareto optimal set extracted from the RSM with (a) $i_{g e n 0}=40$ and $N=30$ (b) $i_{\text {gen } 0}=$ 400 and $N=20$

A statistical approach, with a sample size of 50, was taken to determine the differences in the Pareto optimal set from 1200 evaluations using the two configurations outlined above $\left(i_{g e n 0}=40, \mathrm{~N}=30\right.$ and $\left.i_{\text {gen } 0}=400, \mathrm{~N}=20\right)$. Hypervolume was calculated for each instance of the optimisation using a reference position in the objective space of $(1,1,1)$. The distance measured in the drag rise dimension was the distance to $1-\mathrm{M}_{\mathrm{DR}}$ rather than $\mathrm{M}_{\mathrm{DR}}$ so that the maximal hypervolume indicates the preferred solution. Through the use of an enlarged zeroth generation of $i_{\text {gen } 0}=$ 400, a 0.0011 higher median hypervolume after 1200 evaluations was achieved relative to a smaller initial population of $i_{g e n 0}=40$ (Figure 12). There was also considerably less scatter in the results of the analyses which used $i_{g e n} 0=400$, with a range of 0.0044 compared to a range of 0.0101 for the cases with $i_{\text {gen } 0}=40$, which indicates a more robust approach for the same computational expense.

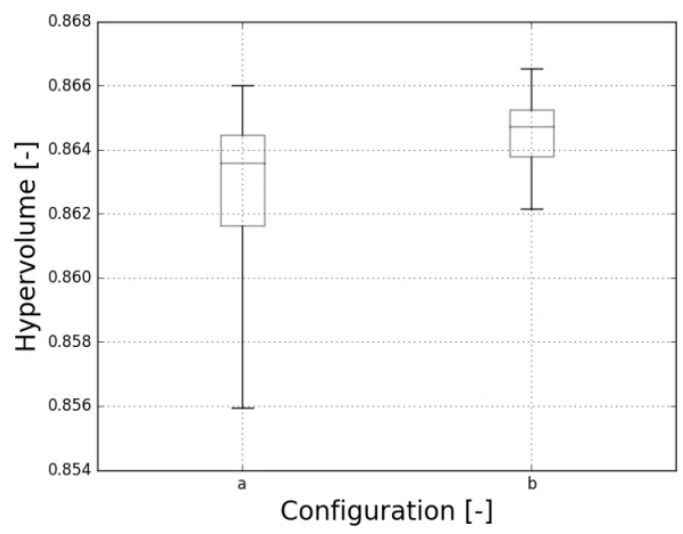

Figure 12. Hypervolume median, IQR and range of final generation for 50 optimisation computations with 1200 evaluations each with (a) $i_{\text {gen } 0}=40$ and $\mathrm{N}=30$ (b) $i_{\text {gen } 0}=400$ and $\mathrm{N}=20$

\section{Influence of constraints}

Often in a MOO problem it is desirable to focus the results to a specific region of interest. For example in a nacelle design optimisation it is typically desired that $\mathrm{M}_{\mathrm{DR}}$ be 0.02 above the cruise Mach number [23]. By applying constraints to the objective functions, it is possible to restrict the objective space which is being resolved and therefore focus the population into a region of interest. This can then reduce the computational cost from finding the entire Pareto front when a more time consuming optimisation procedure incorporating CFD analyses is being performed. An analysis was carried out with a constraint of $\mathrm{M}_{\mathrm{DR}} \geq 0.87$ which produced a denser population in the 
region of interest (Figure 13) with a diversity uniformity of $\Delta=0.443$ signifying no loss in uniformity by adding the constraint.
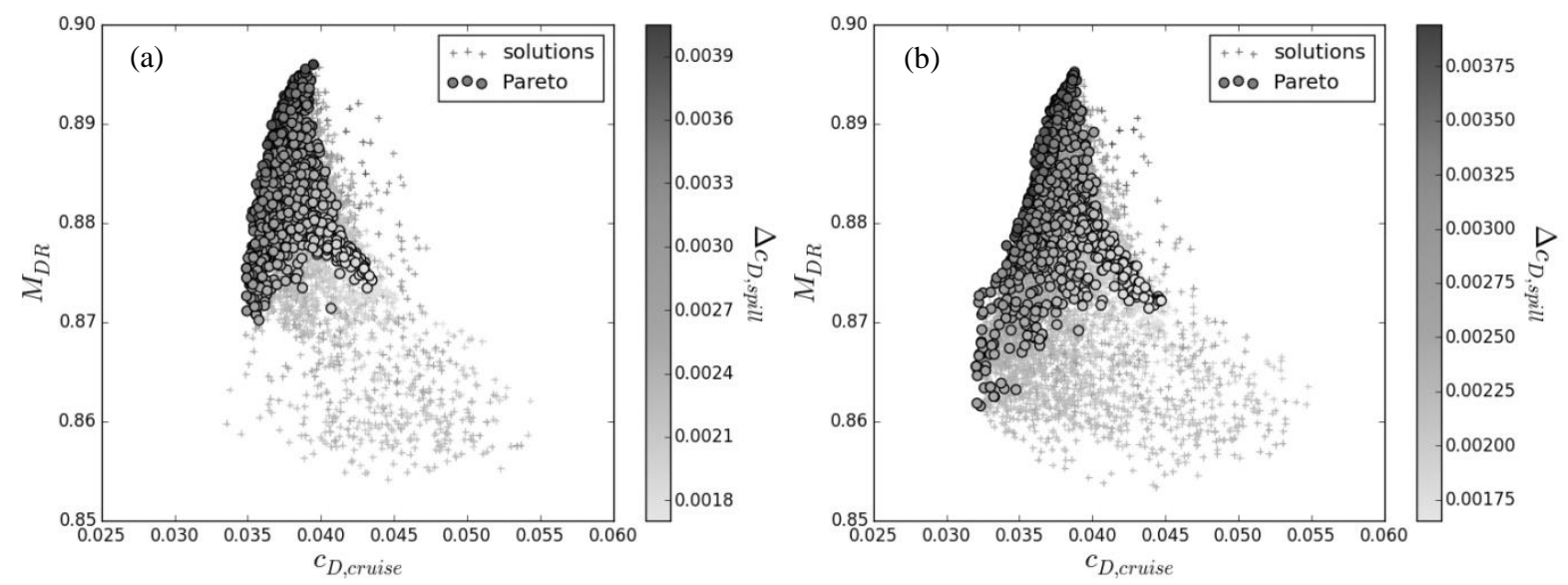

Figure 13. Final Pareto optimal set extracted from the RSM with (a) constrained to $M_{D R} \geq 0.87$ (b) unconstrained

3. Influence of weighting the objective functions

In some situations one objective function is more important to the designer than others. For example, in a nacelle design activity it may be desired to put a greater importance on the cruise drag coefficient over the spillage drag since the majority of the fuel burn is typically dictated by the cruise drag. In these cases, the option to guide the optimisation into regions of particular interest would be desirable. Several methods of applying weights to the objective functions have been proposed [24] [25] [26] and this section deals with applying some to the nacelle RSM optimisation to determine their efficacy.

Objective weightings for evolutionary algorithms can broadly be split into two groups: those which manipulate the dominance function and those which skew the distance algorithm [25]. Different benefits and downsides have been observed for these two approaches with guided dominance for instance being significantly faster [25]. For the purposes of this analysis two approaches have been compared: dominance weighting [24] and distance weighting [26]. Adjustment of the dominance function involves redefining the concept of domination [24]. In a standard implementation of NSGA-II, dominance is defined by the equation (3) in which the inequality must be strictly true in at least one objective. The weighted dominance approach which was utilised skews this definition to include a larger region in at least one objective dimension. This has the impact that if a solution dominates another in the weighted objective then it is allowed to be slightly worse in another. The domination equation then becomes equation (12), where ' $x$ ' dominates ' $y$ ' if the equation is satisfied for all objectives and the inequality is strictly true in at least one. Values of $a_{12}, a_{13} \ldots a_{m(m-1)}$ can then be set to any value to give a weighting of one objective function over another. For instance a value of $a_{12}=2$ would give a preference of the second objective function over the first objective function of 2 units.

$$
\begin{aligned}
& \Omega_{i}(f(x)) \leq \Omega_{i}(f(y)) \\
& \text { where, } \Omega_{1}\left(f_{1}, f_{2}\right)=f_{1}+a_{12} f_{2} \\
& \Omega_{2}\left(f_{2}, f_{1}\right)=f_{2}+a_{21} f_{1} \\
& \Omega_{1}\left(f_{1}, f_{3}\right)=f_{1}+a_{13} f_{3} \ldots \text { etc. }
\end{aligned}
$$

The weighted dominance operator was implemented into the NSGA-II algorithm and tested on the nacelle RSM to determine the performance of this method to find a specific region of the design space. In this instance a lower cruise drag was desired at the expense of the other objectives. For these purposes values of $a_{21}=2, a_{31}=2$, $a_{12}=0.5, a_{13}=0.5, a_{23}=1$ and $a_{32}=1$ were used. This will give the first function $\left(c_{D, \text { cruise }}\right)$ a weighting over the other two $\left(M_{D R}, \Delta c_{D, \text { spill }}\right)$ and make the other two equally weighted between each other. These settings were used for an analysis to optimise the three objective functions in the nacelle RSM. The results of this (Figure 14) demonstrated that a weighted dominance operator can give improved values of a particular objective function at the expense of the others. Specifically, $c_{D, \text { cruise }}$ was reduced to 0.0322 , compared to 0.0330 in the unweighted example. 
However maximum drag rise Mach number decreased from 0.896 to 0.895 due to the weighting and minimum $\Delta c_{D, \text { spill }}$ remained the same as the unweighted optimisation at 0.0017 .
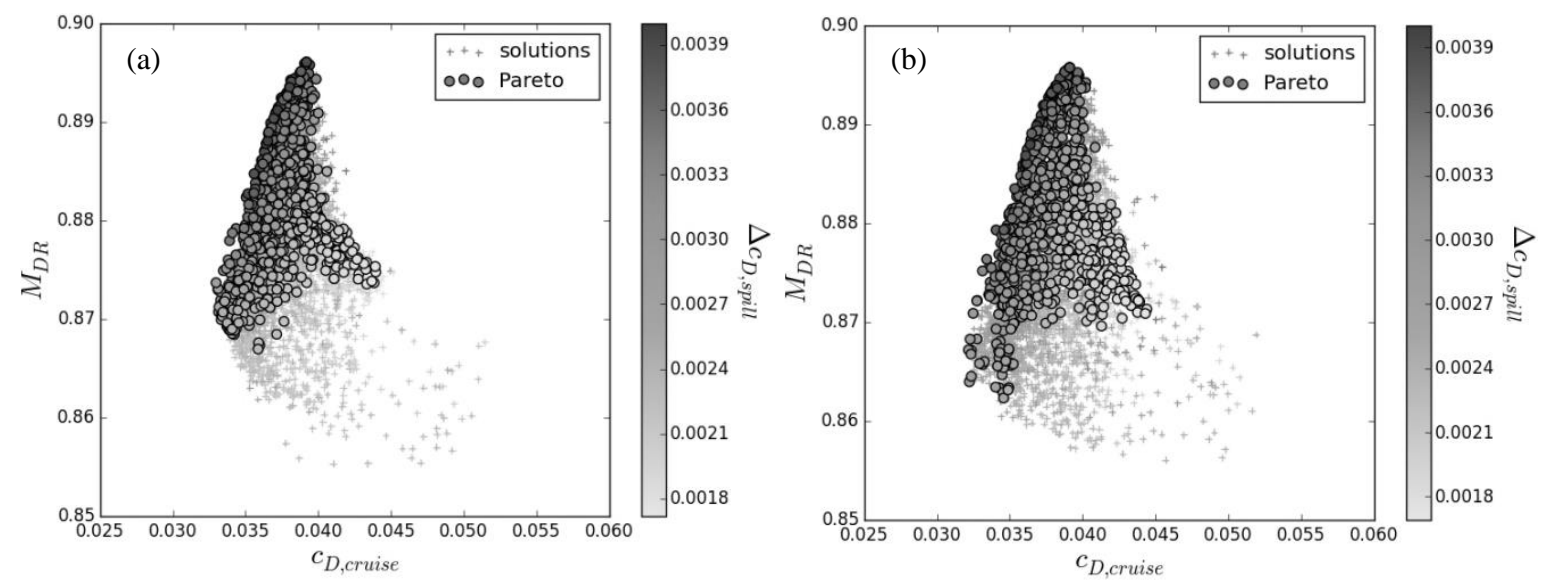

Figure 14. Final Pareto optimal set extracted from the RSM with (a) unweighted (b) weighted through dominance operator

An alternative means of weighting the objective functions in the optimisation is to adjust the distance operator [26]. In the standard implementation, the Euclidean distance is calculated between each individual in a NDS and used to determine the most isolated individuals. In a weighted distance operator, the Euclidean distance is skewed to give higher values to individuals which perform better in a preferred objective function whilst still including information on the spread of the individuals (equation (13)).

$$
\begin{gathered}
d(i, j)=\left[\sum_{k=1}^{m} w_{k}^{\prime} \frac{\left(f_{k}^{i}-f_{k}^{j}\right)^{2}}{\left(f_{k}^{m a x}-f_{k}^{m i n}\right)^{2}}\right]^{\frac{1}{2}} \\
\text { where } w_{k}^{\prime}=\frac{\left(1-w_{k}\right)}{\max _{m=1}^{m=n}\left(1-w_{m}\right)} \text { for convex pareto fronts } \\
\text { or } w_{k}^{\prime}=\frac{w_{k}=\frac{m_{k}^{m=n} w_{m}}{\max _{m=1}^{m=n} w_{m}} \text { for non-convex pareto fronts }}{w_{k} \in(0,1)}
\end{gathered}
$$

To compare to the performance of dominance weighting, a similar problem was posed for distance weighting. To weight cruise drag over spillage drag and drag rise Mach number values of $w_{k}^{\prime}=1, w_{2}^{\prime}=0.5$ and $w_{3}^{\prime}=0.5$ were used. An analysis was carried out with these settings for the three objective functions used in the nacelle RSM. 4000 evaluations were undertaken over 100 generations using the settings in Table 1 . The results of this analysis (Figure 15) demonstrated that the use of a weighted distance operator provided more beneficial values of a particular objective function at the expense of the others. In this case $c_{D, \text { cruise }}$ was reduced to 0.0318 , compared to 0.0330 in the unweighted example. However, maximum drag rise Mach number decreased from 0.896 to 0.895 due to the weighting whilst minimum $\Delta c_{D, \text { spill }}$ was unchanged. 

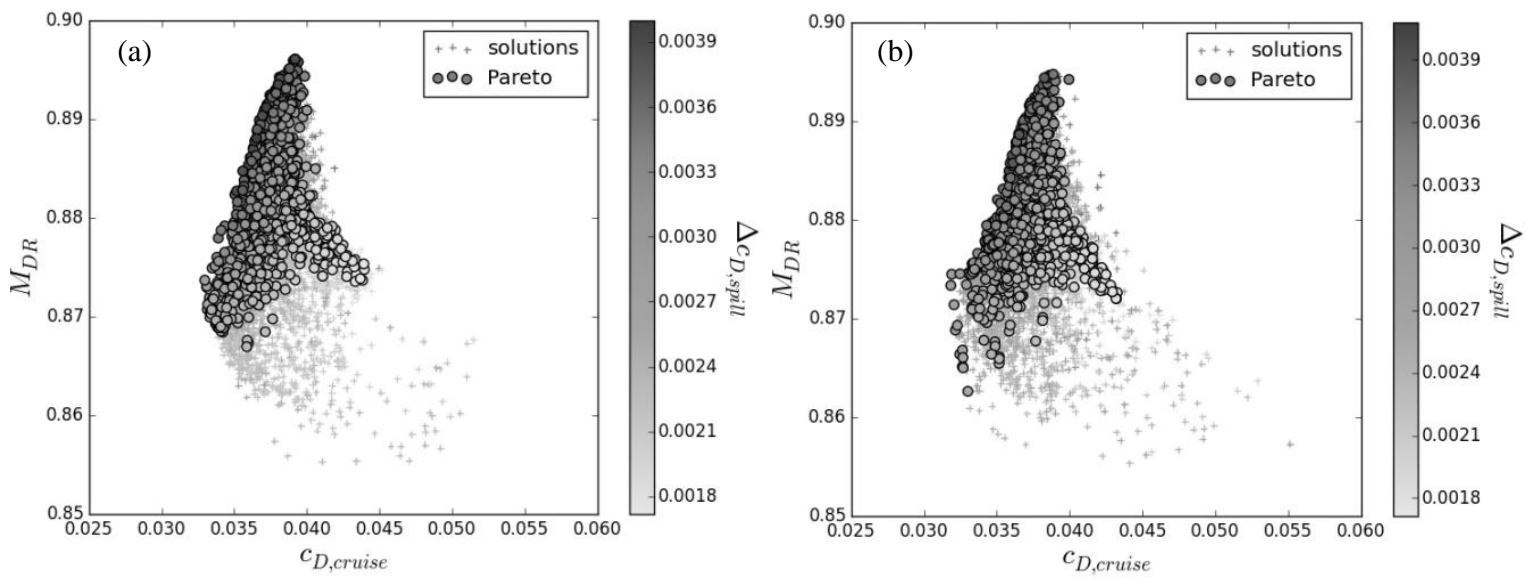

Figure 15. Final Pareto optimal set extracted from the RSM with (a) unweighted (b) weighted through spacing operator

To compare the two different weighting approaches, the mean values of the cruise drag objective function were assessed throughout the generations. The mean value of $c_{D, \text { cruise }}$ in the final Pareto set from the unweighted optimisation was 0.0376 . When weighting was applied to the spacing operator (13) this beneficially decreased to 0.0373 , however when weighting was applied to the dominance operator (12) this value adversely increased to 0.0379. The application of objective weighting to the spacing operator began to clearly bias the solutions found to the chosen objective after eight generations (Figure 16) which suggests it is more applicable in this RSM optimisation to explore specific regions of the design space. This is just one implementation of the dominance weighting however and it is possible that the non-deterministic nature of the algorithm has simply given a poor result this time. It is also possible that the weighting factors could be more finely tuned with additional analysis, however since there are more factors in the dominance weighting than in the spacing weighting, this would take more computational effort.

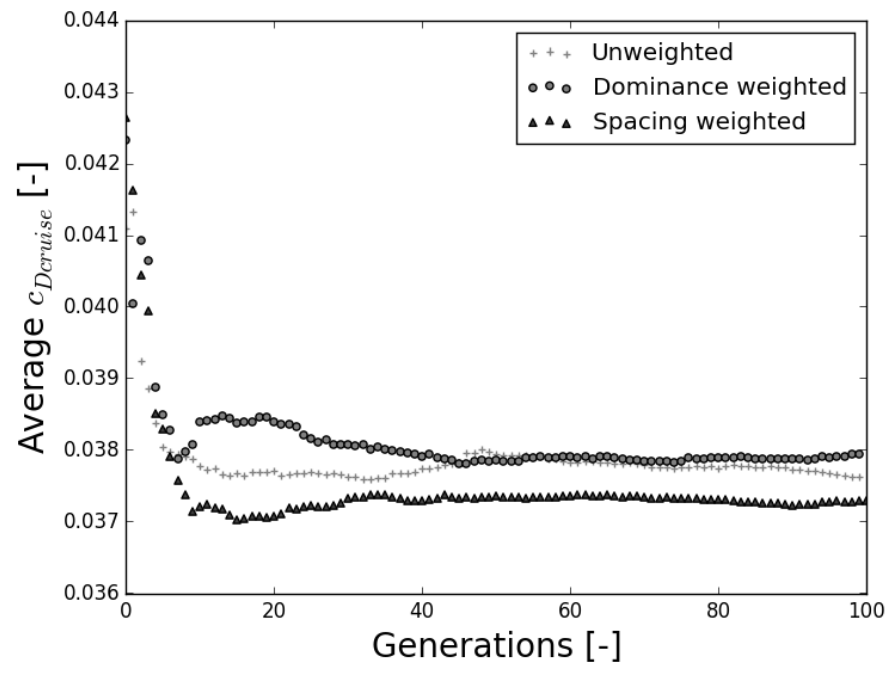

Figure 16 Mean $c_{D, \text { cruise }}$ of Pareto set as a function of generation for different objective weighting configurations 


\section{Conclusions}

A method has been demonstrated to assess the design space for nacelle performance at a preliminary stage of the design. The optimisation method has been demonstrated to converge to the Pareto fronts of both the 2 objective Kursawe problem [17] and the 3 objective DTLZ2 problem [15] with a convergence of $M_{1}^{*}=0.0109$ for DTLZ2. The method was applied to an RSM of nacelle performance to optimise for three objective functions. This method produced a three dimensional Pareto front which can be used in preliminary design to ensure good performance at both on and off design conditions. To assess the robustness of the method, different initial sample sizes were tested. It was shown that over 1200 evaluations a better converged Pareto set can be obtained from using an initial sample size of 400 and 19 subsequent generations than using 30 generations of 40 . This provided on average a higher hypervolume with less scatter for the same number of evaluations. Application of a constraint to one objective function has also been shown to have little impact on the diversity of the final solution but can provide a better use of resources. Two methods of weighting the objective functions have also been demonstrated and distance weighting shown to provide a more efficient bias towards a particular objective for these cases.

\section{Acknowledgments}

The lead author of this paper was partially supported by a grant from the Engineering and Physical Sciences Research Council. The authors would like to thank Rolls Royce plc for their support in this project.

\section{References}

1. Daly M. (ed.), Jane's Aero-Engines, $28^{\text {th }}$ ed., IHS Global Ltd, Surrey, 2010, p. 64

2. Parker R., "From blue skies to green skies: engine technology to reduce the climate-change impacts of aviation", Technology Analysis \& Strategic Management, Vol. 21, No. 1, 2009, pp. 61-78

3. Hoheisel H., "Aerodynamic Aspects of Engine-Aircraft Integration of Transport Aircraft", Aerospace Science and Technology, Vol 1, No. 7, 1997, pp. 475-487

4. Stankowski T. P., MacManus D. G., Sheaf C. T., and Grech N., "Aerodynamic interference for aero-engine installations", 54 $4^{\text {th }}$ AIAA Aerospace Sciences Meeting, AIAA SciTech, Washington, AIAA 2016-0766, DC, 2016

5. Daggett D. L., Brown S. T., and Kawai R. T., "Ultra-Efficient Engine Diameter Study”, NASA, CR-2003-212309, 2003

6. Song W., and Keane A., "Surrogate-Based Aerodynamic Shape Optimization of a Civil Aircraft Engine Nacelle", AIAA Journal, Vol. 45, No. 10, 2007, pp. 2565-2574

7. Toubin H., Salah El Din I., and Meheut M., "Multipoint Aerodynamic High Fidelity Shape Optimization of an Isolated Engine Nacelle", 52 ${ }^{\text {nd }}$ AIAA Aerospace Sciences Meeting, AIAA SciTech, AIAA 2014-0903, Washington, DC, 2014

8. Farokhi S., Aircraft Propulsion, $1^{\text {st }}$ ed., Wiley, Chichester, England, 2009, p.241

9. Schaffer J. D., "Multiple Objective Optimization with Vector Evaluated Genetic Algorithms", Proceeding of the ${ }^{\text {st }}$ International Conference on Genetic Algorithms, Hillsdale, NJ, 1985, pp. 93-100

10. Heidebrecht A., Stankowski T., and MacManus D., "Parametric Geometry and CFD Process for Turbofan Nacelles", Proceedings of ASME 2016 Turbo Expo: Turbomachinery Technical Conference and Exposition, GT2016-57784, New York, 2016

11. Christie R., Heidebrecht A., and MacManus D., "An Automated Approach to Nacelle Parameterisation Using Intuitive Class Shape Transformation Curves", Proceedings of ASME 2016 Turbo Expo: Turbomachinery Technical Conference and Exposition, GT2016-57849, New York, 2016

12. Kulfan B. M., and Bussoletti J. E., ““'Fundamental” Parametric Geometry Representations for Aircraft Component Shapes", 11th AIAA/ISSMO Multidisciplinary Analysis and Optimization Conference: The Modeling and Simulation Frontier for Multidisciplinary Design Optimization, AIAA—2006-6948 Washington, DC, 2006

13. Roskam J., and Lan C.-T.E., Airplane Aerodynamics and Performance, $3^{\text {rd }}$ ed., DARcorporation, Lawrence, KA, 1997, p. 141

14. Deb K.., Pratap A., Agarwal S., and Meyarivan T., "A Fast and Elitist Multiobjective Genetic Algorithm: NSGA-II", IEEE Transactions on Evolutionary Computation, Vol. 6, No. 2, 2002, pp. 182-197

15. Deb K., Thiele L., Laumanns M., and Zitzler E., "Scalable Test Problems for Evolutionary Multi-objective Optimization", Institut fur Tecnische Informatik und Kommunikationsnetze, ETH Zurich, TIK-Technical Report No. 112, Zurich, 2001

16. Zitzler E., Deb K., and Thiele L., "Comparison of Multiobjective Evolutionary Algorithms: Empirical Results, Evolutionary Computation, Vol. 8, No. 2, 2000, pp. 173-195 
17. Kursawe F., "A variant of evolution strategies for vector optimization”, Parallel Problem Solving from Nature Proceedings of the $1^{\text {st }}$ Workshop, PPSN1, Berlin, 1991, pp. 193-197

18. Zitzler E. and Thiele L., "Multiobjective Optimization Using Evolutionary Algorithms - A Comparative Case Study", Parallel Problem Solving from Nature Proceedings of the $5^{\text {th }}$ Workshop, PPSN V, Berlin, 1998, pp. 292-301

19. Deb K., Multi-Objective Optimization Using Evolutionary Algorithms, ${ }^{\text {st }}$ ed., Wiley, Chichester, England, 2001, p.333

20. Nebro A. J., Luna F., and Alba E., "New Ideas in Applying Scatter Search to Multiobjective Optimization", Evolutionary Multi-Criterion Optimization: Third International Conference, edited by Coello C.C., Hernandez A., Guanajuatom, Mexico, 2005, pp. 443-458

21. Xie D., Ding L., Hu Y., Wang S., Xie C., and Jiang L., “A multi-algorithm balancing convergence and diversity for multi-objective optimization”, Journal of Information Science and Engineering, Vol. 29, No. 5, 2013, pp. 811-834

22. Bringmann K., Friedrich T., Neumann F., and Wagner M., "Approximation-Guided Evolutionary Multi-Objective Optimization", Proceedings of the Twenty-Second International Jount Conference on Artifical Intelligence, Menlo Park, CA, 2011, pp. 1198-1203

23. Borradaile J. A., "Towards the optimum ducted UHBR engine", AIAA/AHS/ASME/ASEE $24^{\text {th }}$ Joint Propulsion Conference, AIAA-88-2954, Washington, DC, 1988

24. Branke J., Kaußler T., and Schmeck H., "Guidance in evolutionary multi-objective optimization", Advances in Engineering Software, Vol. 32, No. 6, 2001, pp. 499-507

25. Branke J., and Deb K., "Integrating User Preferences into Evolutionary Multi-Objective Optimization”, Indian Institute of Technology, Technical Report KanGAL Report Number 2004004, Kanpur, 2004

26. Deb K., Multi-Objective Optimization Using Evolutionary Algorithms, $1^{\text {st }}$ ed., Wiley, Chichester, England, 2001, pp.393-396 
2017-01

\section{An optimization method for nacelle design}

\section{Robinson, M.}

American Institute of Aeronautics and Astronautics

Matthew H. Robinson, David G. MacManus, Alexander Heidebrecht, and Nicholas Grech. "An optimization method for nacelle design", 55th AIAA Aerospace Sciences Meeting, AIAA SciTech Forum, (AIAA 2017-0708)

https://dspace.lib.cranfield.ac.uk/handle/1826/11409

Downloaded from Cranfield Library Services E-Repository 